\title{
Conclusiones de la primera mesa de trabajo en diabetes
}

Este texto está basado en un documento que presenta las memorias de la primera mesa de trabajo en diabetes, realizada en 2020 por la Asociación Colombiana de Endocrinología, Diabetes y Metabolismo (ACE), y que ha sido replicado por varios medios de comunicación.

\section{Doctor}

Enrique Ardila

Editor

Revista Colombiana de Endocrinología, Diabetes y Metabolismo

Apreciado Dr. Ardila:

- Según la Federación Internacional de Diabetes, aproximadamente 463 millones de personas en el mundo viven con diabetes (1), y casi el $7 \%$ de los colombianos también han sido diagnosticados con esta patología (2).

- $\quad$ En Colombia, durante 2019, solo el 51,08 \% de los 1,3 millones de pacientes del sistema de salud podían controlar la hemoglobina glucosilada $\left(\mathrm{HbA}_{1 \mathrm{c}}\right)$, lo cual constituye una señal preocupante, ya que la falta de control de esta proteína se ha relacionado con otras afecciones como eventos cerebrovasculares (3).

La diabetes es una condición con alta prevalencia en el mundo y en Colombia. En efecto, casi el 7 \% de la población de nuestro país ha sido diagnosticada con esta patología y cerca de 17000 muertes se asocian a ella anualmente (4). Si bien el panorama muestra la importancia de la toma de decisiones, existen herramientas y tecnologías emergentes que facilitan los procedimientos, como la monitorización instantánea de glucosa, el tiempo en rango (Time-in-Range, TIR) y la telemonitorización.

En la primera mesa de trabajo en diabetes, realizada en 2020 por la Asociación Colombiana de Endocrinología, Diabetes y Metabolismo (ACE), los expertos discutieron temas importantes sobre el control de esta enfermedad en Colombia. Una de ellas fue la Dra. Karen Feriz, endocrinóloga, quien concluyó que las tasas de mortalidad en personas con diabetes se asocian principalmente con eventos cardiovasculares. Sin embargo, la irrupción de nuevas terapias y estrategias de seguimiento ha logrado ofrecer reducciones significativas.

De otro lado, la mortalidad por diversas causas como sepsis, infecciones respiratorias, complicaciones por amputaciones, enfermedades hepáticas y renales ha aumentado en la población con diabetes; todas estas condiciones se relacionan con la falta de un control glucémico adecuado. Asimismo, se estima que el 57,1 \% (4) de las personas con esta patología en Colombia no controlan su enfermedad, lo cual puede estar relacionado, entre otras cosas, con la falta de educación sobre el tema.

Otro experto de la ACE, el Dr. José Fernando Botero, internista endocrinólogo, mencionó que algunos de los desafíos para el cuidado de la diabetes son la falta de comunicación entre el médico y el paciente, el bajo acceso a nuevas herramientas de monitorización, la necesidad de realizar análisis y la educación. Con estos elementos, se puede efectuar la implementación adecuada de un modelo de atención médica que incluya la tecnología.

En cuanto a la innovación en el campo, existen herramientas como la monitorización instantánea de glucosa, una tecnología que mide los niveles de glucosa sin pinchazos y permite obtener información completa y actualizada sobre el nivel de glucosa de una persona. Esta ofrece diferentes métricas que contribuyen a evaluar el nivel de control que el paciente tiene. Dentro de dichas herramientas se destaca el TIR, un estándar de control que muestra el porcentaje de tiempo diario que una persona pasa con sus niveles de glucosa en un rango óptimo. Con el tiempo, dicho instrumento proporciona más evidencia, apoyo y aprobación para su uso en múltiples asociaciones científicas y entidades reguladoras.

Recientemente, se agregó la telemonitorización como parte de la ecuación, lo que ha permitido optimizar el proceso de control glucémico. Toda esta tecnología estableció un conjunto de herramientas que facilitan el autocontrol, la toma de decisiones, la autonomía y conectan a los médicos con los usuarios, a fin de mantener un canal de comunicación basado en datos actualizados.

El principal beneficio de adoptar tecnologías como la monitorización instantánea de glucosa es que se puede reducir la hemoglobina glucosilada (HbA1c), así como minimizar los eventos de hipoglucemia y aumentar el TIR. Así se muestra en un estudio de Real World Data basado en el uso de la he- 
rramienta tecnológica de detección de glucosa, en el cual se observa una reducción del $49 \%$ en los tiempos de hipoglucemia y un aumento del $40 \%$ en el TIR (5). Esto se traduce en comodidad y calidad de vida.

La monitorización instantánea de glucosa tiene una trayectoria comprobada en cambiar vidas y en mejorar los resultados de salud para las personas con diabetes. Creemos que esto se debe a la gran cantidad de datos en tiempo real que proporciona el sistema. La tecnología ayuda a visualizar los datos y las tendencias de la glucosa y puede ayudar a los enfermos con diabetes a hacer los ajustes necesarios para garantizar estar óptimamente dentro del TIR. Estos datos también proporcionan información procesable que permite tener conversaciones más significativas con los profesionales de la salud, a fin de conseguir un tratamiento óptimo.

Aunque Colombia ha progresado en el acceso e implementación de tecnologías emergentes para el cuidado de la diabetes, todavía hay algunos pasos por seguir, como la actualización de las guías nacionales de práctica clínica, las cuales deberían incluir nuevos estándares para su control (entre ellos, el TIR), y también la ruta para acceder a nuevas tecnologías como la monitorización instantánea de glucosa, teniendo en cuenta la caracterización del paciente.
Asimismo, "La educación es un factor clave para el éxito en la implementación de estas tecnologías y tiene un impacto directo en la calidad de vida de los pacientes y la sostenibilidad del sistema de salud. Por esa razón, se necesita un mecanismo que integre tecnología y los programas de educación del paciente que permitan el autocuidado y el uso de herramientas que también optimicen la vida cotidiana de las personas que viven con diabetes “, dijo el Dr. Oswaldo Rincón, internista endocrinólogo y experto de la ACE.

Como un llamado a la acción para los usuarios, la ACE enfatiza el uso de herramientas educativas disponibles y la búsqueda autónoma de guías de práctica clínica para aprovechar las soluciones emergentes que están dando forma al futuro de la diabetes y la salud.

Cordialmente,

Henry Tovar Cortés, MD

Médico internista, endocrinólogo

Presidente de la Asociación Colombiana de Endocrinología,

Diabetes y Metabolismo (ACE)

\section{Referencias}

1. International Diabetes Federation [internet]. Diabetes facts \& figures [actualizada el 12 de febrero de 2020]. Disponible en: https://www.idf.org/ aboutdiabetes/what-is-diabetes/facts-figures.html

2. Asociación Colombiana de Endocrinología [internet]. Bogotá D.C: marzo de 2020 [consultada el 24 de agosto de 2020] Diabetes y COVID-19 Cuidados especiales para personas con diabetes en época de coronavirus. Disponible en: https://www.endocrino.org.co/wp-content/uploads/2020/03/Recomendaciones-ACE-sobre-Diabetes-y-Covid-19.pdf

3. Cuenta de alto costo [internet]. Situación de la enfermedad renal crónica, la hipertensión arterial y la diabetes mellitus en Colombia 2019; mayo de 2020. Disponible en: https://cuentadealtocosto.org/site/wp-content/uploads/2020/06/CAC.Co_2020_06_24_Libro_Sit_ERC_2019V7.pdf
4. Hospital Universitario San Ignacio [internet]. La Diabetes, la causa de 17 mil muertes en Colombia; 12 de noviembre de 2019 [consultada el 24 de agosto de 2020]. Disponible en: http://www.husi.org.co/el-husi-hoy/ladiabetes-la-causa-de-17-mil-muertes-en-colombia

5. Dunn T. Evidence of a strong association between frequency of flash glucose monitoring and glucose control measures during real-world usage, E-poster presented. En: the 10th International Conference on Advanced Technologies \& Treatments for Diabetes (ATTD 2017); París: 15-18 febrero de 2017. 


\section{JUXTAPID ES EFECTIVO EN LA DISMINUCIÓN DE C-LDL PARA LOGRAR METAS}
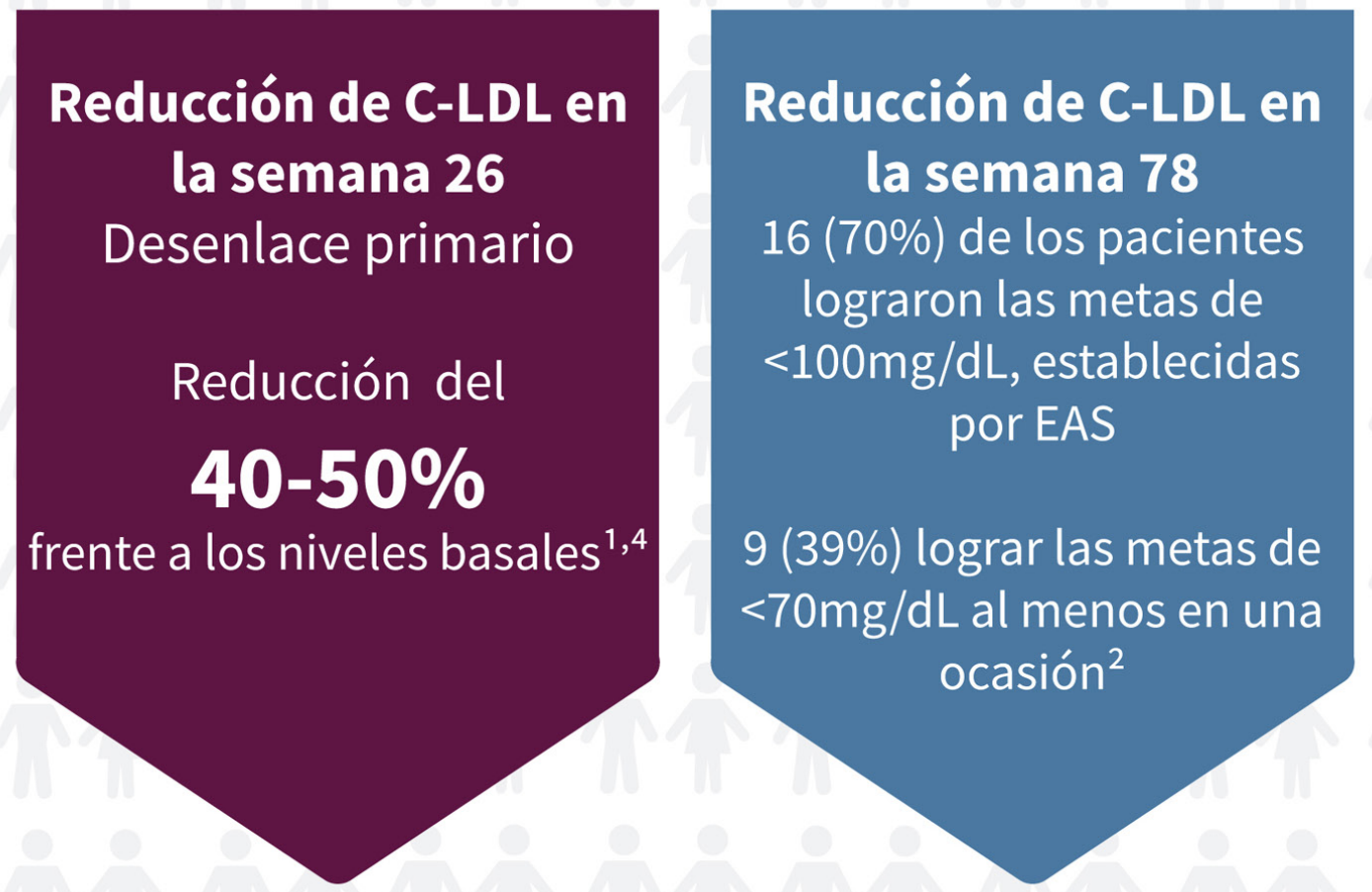

Juxtapid fue evaluado en un ensayo clínico de fase 3, de un solo brazo, durante 78 semanas donde 29 pacientes adultos con Hipercolesterolemia Familiar Homocigota (HFHO) fueron tratados con Juxtapid como tratamiento principal, incluyendo otras terapias complementarias (en el 63\% de los pacientes) ${ }^{1}$

- La dosis de Juxtapid fue escalada en base a la tolerabilidad de los pacientes (5mg-60mg/día) $)^{1}$

- El criterio de valoración principal del estudio fue la reducción porcentual de los niveles de C-LDL en la semana $26^{1}$

\section{Después de 78 semanas, los pacientes pudieron ingresar a una extensión del estudio y recibieron tratamiento durante 5.7 años $^{3}$}

